\title{
Dyslipidemia treatment of patients with diabetes mellitus in a US managed care plan: a retrospective database analysis
} Peter P Toth* ${ }^{* 1,2,3}$, Victoria Zarotsky ${ }^{4}$, Jane M Sullivan ${ }^{4}$ and Dave Laitinen ${ }^{5}$

\author{
Address: ${ }^{1}$ Sterling Rock Falls Clinic, Sterling, IL, USA, ${ }^{2}$ University of Illinois College of Medicine, Peoria, Illinois, USA, ${ }^{3}$ Southern Illinois \\ University School of Medicine, Springfield, Illinois, USA, ${ }^{4}$ i3 Innovus, Eden Prairie, Minnesota, USA and ${ }^{5}$ Abbott Laboratories, \\ Abbott Park, Illinois, USA \\ E-mail: Peter P Toth* - peter.toth@srfc.com; Victoria Zarotsky - victoria.zarotsky@i3innovus.com; \\ Jane M Sullivan - jane.sullivan@i3innovus.com; Dave Laitinen - dave.laitinen@abbott.com \\ ${ }^{*}$ Corresponding author
}

Published: 18 May 2009

Cardiovascular Diabetology 2009, 8:26 doi: 10.1186/1475-2840-8-26

This article is available from: http://www.cardiab.com/content/8/I/26

(C) 2009 Toth et al; licensee BioMed Central Ltd.

This is an Open Access article distributed under the terms of the Creative Commons Attribution License (http://creativecommons.org/licenses/by/2.0), which permits unrestricted use, distribution, and reproduction in any medium, provided the original work is properly cited.
Received: II March 2009

Accepted: 18 May 2009

\begin{abstract}
Background: To evaluate real-world pharmacologic treatment of mixed dyslipidemia in patients with diabetes mellitus (DM).

Methods: All commercial health plan members in a large US managed care database with complete lipid panel results (HDL-C, LDL-C, TG) between 1///2006 and 12/31/2006 were identified $(N=529,236)$. DM patients $(N=53,679)$ with mixed dyslipidemia were defined as having any 2 suboptimal lipid parameters $(N=28,728)$. Lipid treatment status 6 months pre- and postindex date was determined using pharmacy claims for any lipid therapy.

Results: Post-index, $41.1 \%$ of DM patients with 2 abnormal lipid parameters and $45.1 \%$ with 3 abnormal lipid parameters did not receive lipid-modifying treatment. Post-index treatment rates were $57.4 \%, 63.6 \%$, and $66.4 \%$ for patients with LDL-C, HDL-C, and TG in the most severe quartiles, respectively. Statin monotherapy was the primary lipid-modifying regimen prescribed ( $54.8 \%$ and $47.8 \%$ of patients with any 2 and all 3 lipids not at goal, respectively). Less than $30 \%$ of treated patients received combination therapy.

Conclusion: Over $40 \%$ of DM patients with mixed dyslipidemia received no lipid-modifying therapy during the follow-up period. Those who were treated were primarily prescribed statin monotherapy. This study suggests that DM patients are not being treated to ADA-suggested targets.
\end{abstract}

\section{Introduction}

Cardiovascular disease (CVD) is a primary cause of morbidity and mortality among patients with hyperglycemia or type 2 diabetes mellitus (DM) despite the availability of effective therapies to treat major risk factors such as elevated blood pressure and cholesterol levels [1,2]. The most common lipid pattern in patients with DM, hypertension, and/or the metabolic syndrome includes hypertriglyceridemia, increased concentrations of small dense low-density lipoprotein particles, low levels of highdensity lipoprotein cholesterol (HDL-C), increased remnant lipoproteins, and elevated apolipoprotein B concentrations $[3,4]$. This profile of mixed dyslipidemia significantly increases risk for all forms of atherosclerotic disease, including coronary heart disease (CHD) [5-8].

The burgeoning prevalence of insulin resistance throughout the world is greatly increasing the incidence of mixed 
dyslipidemia. It is estimated that approximately $40 \%$ of patients with coronary artery disease have low-density lipoprotein cholesterol (LDL-C) levels below $130 \mathrm{mg} / \mathrm{dL}$ yet these patients also have low levels of HDL-C, with or without increased levels of triglycerides (TG) $[9,10]$. Overall, isolated low HDL-C affects $20 \%$ to $30 \%$ of patients with CHD, representing several million people in the United States $[9,10]$.

Observational studies indicate that low HDL-C levels are strongly and independently associated with increased CHD risk [11-13]. The results of the first major clinical trial specifically focusing on the treatment of low HDL-C demonstrated that lipid treatment that raised HDL-C and lowered TG but had no effect on LDL-C substantially reduced the incidence of major cardiac and cerebrovascular events [14].

Elevated serum levels of TG are an independent risk factor for CHD even after adjustment for HDL-C [7]. In a subanalysis of the Pravastatin or Atorvastatin Evaluation and Infection Therapy-Thrombolysis In Myocardial Infarction 22 (PROVE-IT TIMI 22) trial to assess the impact of low on-treatment TG on CHD risk beyond LDL-C $<70 \mathrm{mg} / \mathrm{dL}$, Miller et al. [15] demonstrated that on-treatment TG below $150 \mathrm{mg} / \mathrm{dL}$ was independently associated with a lower risk of recurrent CHD events. The Veterans Affairs HDL Intervention Trial (VA-HIT), which included patients with low HDL-C (mean $31 \mathrm{mg} / \mathrm{dL}$ ) and LDL-C (mean $111 \mathrm{mg} / \mathrm{dL}$ ), found that patients receiving gemfibrozil had a $22 \%$ relative risk reduction in the primary end point of time to first nonfatal myocardial infarction or CHD death $(95 \%$ CI $7 \%$ to $35 \%$, p = $0.006)$. There was also a significant reduction in cerebrovascular events. Among 627 patients with DM, there was a $24 \%$ relative risk reduction for the expanded end point (CHD-related death, nonfatal myocardial infarction, or definite stroke). Likewise, among 1,449 patients with $\geq 3$ criteria that define the metabolic syndrome (impaired fasting glucose; hypertension; obesity; high TG; or low HDL-C), the relative risk reduction with gemfibrozil was an even more impressive $35 \%$ for the expanded end point [14]. Together with data from the Helsinki Heart Study [16] and a subgroup analysis from the Bezafibrate Infarction Prevention study [17], these data suggest that fibrates may be particularly effective in treating dyslipidemia in patients with the metabolic syndrome.

Multiple guidelines writing groups advocate combination therapy for the management of multiple lipid abnormalities (NCEP ATPIII, ADA, AHA/ACC) $[18,19]$. Statin-fibrate combination therapy has been found to be more successful than statin monotherapy in achieving therapeutic targets in dyslipidemic patients with DM or the metabolic syndrome [20-22]. The ongoing Action to Control Cardiovascular Risk in Diabetes (ACCORD) trial was designed to evaluate the effect of simvastatinfenofibrate combination therapy on cardiovascular risk in patients with type 2 DM [23]. The effect of statinniacin combination therapy on atherosclerosis progression in patients with high cardiovascular risk (CHD and low HDL-C levels) has been evaluated in both the Arterial Biology for the Investigation of the Treatment Effects of Reducing Cholesterol 2 (ARBITER 2) and the HDL Atherosclerosis Treatment (HATS) studies. These trials demonstrated that the use of statin-niacin combination therapy is associated with stabilization of atherosclerotic disease.

Despite the abundance of data confirming the role of suboptimal lipoprotein levels as a risk factor for CHD and the availability of guidelines enumerating therapeutic lifestyle modifications and pharmaceutical treatment options for the management of mixed dyslipidemia, a substantial proportion of dyslipidemic patients remain untreated or inappropriately treated $[24,25]$, or patients choose to discontinue treatment soon after it is initiated [26]. The primary objective of the present study was to achieve greater understanding of treatment patterns in "real-world" patients by evaluating pharmacologic treatment of DM patients with mixed dyslipidemia enrolled in a commercial health plan. Specifically, the purpose was to determine the proportion of DM patients with suboptimal LDL, HDL-C, and/or TG values not being appropriately treated with lipid-modifying medications.

\section{Methods \\ Data Source}

This was a retrospective claims data analysis using medical and pharmacy data, laboratory results, and enrollment information from a large managed health care plan in the United States, with the largest concentration of patients being in the southern and midwestern regions. Claims for services provided to members of this health plan are submitted for payment by physicians, facilities, and pharmacies. At the time the study was conducted, the administrative claims database included data for approximately 14 million health plan enrollees with both medical and pharmacy benefits. All study data were de-identified and accessed with protocols compliant with the Health Insurance Portability and Accountability Act. Institutional Review Board approval was therefore not required for this study.

\section{Study Subject Identification}

This study was conducted to determine lipid treatment patterns in DM patients with mixed dyslipidemia. Study patients included commercial health plan enrollees with 
a laboratory value for LDL-C, HDL-C, and TG all drawn on the same day during the time period from January 1, 2006 through December 31, 2006. An index date was set as of the date of the first suboptimal test result or first optimal test result. Patients were required to have been continuously enrolled for 182 days prior to and 182 days following the index date.

Two groups of patients were created: a subset with all lipid values (LDL-C, HDL-C, and TG) under control (categorized as the "optimal cohort") and another subset with at least 1 suboptimal lipid value ("suboptimal cohort"). The criteria for the suboptimal cohort were developed in accordance with the National Cholesterol Education Program (NCEP) Adult Treatment Panel (ATP) III [19] and American Heart Association (AHA) guidelines [13-15]. For DM/CHD patients, suboptimal lipid values were defined as LDL-C $\geq 100 \mathrm{mg} / \mathrm{dL}, \mathrm{HDL}-\mathrm{C} \leq$ $40 \mathrm{mg} / \mathrm{dL}$ for males and $\leq 50 \mathrm{mg} / \mathrm{dL}$ for females, and $\mathrm{TG} \geq 150 \mathrm{mg} / \mathrm{dL}$.

\section{Study Measures}

Patient demographic variables (age, gender, and geographic location) were captured from the enrollment data. Lipid lab values (LDL-C, HDL-C, TG) were obtained from the lab results data. Study patients were observed for 182 days prior to the index date (pre-index period) and for 182 days after the index date (post-index period) to determine their lipid risk factors for CVD and their pharmaceutical prescription patterns. For the assignment of LDL-C goal, patients were classified into 4 risk categories: (1) age/gender (male, $\geq 45$ years; female, $\geq 55$ years); (2) CHD (a medical claim indicating presence of CHD during the pre-index period); (3) hypertension (presence of ICD-9-CM code 401.x-404.x, $642.0 \mathrm{x}-642.2 \mathrm{x}, 642.7 \mathrm{x}$ during the pre-index period); and (4) DM (presence of ICD-9-CM code 250.xx or at least 2 filled prescriptions for oral antidiabetic agents during the pre-index period).

The presence of a pharmacy claim for any lipid therapy, including statins, fibrates, niacin, or various combinations, was determined. Specifically, separate determinations were made to identify patients with the following prescription patterns during the pre-index and postindex periods: (1) pharmacy claim for a statin (atorvastatin, cerivastatin, fluvastatin, lovastatin, pravastatin, rosuvastatin, simvastatin, or atorvastatin + amlodipine [Caduet ${ }^{\circledR}$ ]) and without a claim for other lipid-modifying medications; (2) pharmacy claim for a fibrate (clofibrate, gemfibrozil, fenofibrate) and without a claim for other lipid-modifying medications; (3) pharmacy claim for a niacin/nicotinic acid and without a claim for other lipid-modifying medications; (4) pharmacy claim for "other" medication (cholestyramine, colestipol, colesevelam, ezetimibe) and without a claim for other lipid-modifying medications; (5) pharmacy claim for a statin and a fibrate; (6) pharmacy claim for a statin and niacin/nicotinic acid; (7) pharmacy claim for a statin and other lipid-modifying medication; (8) pharmacy claim for a fibrate and a niacin; (9) pharmacy claim for a fibrate and other medications (cholestyramine, colestipol, colesevelam, ezetimibe); (10) pharmacy claim for a niacin and other medications (cholestyramine, colestipol, colesevelam, ezetimibe); (11) combinations other than those already mentioned; and (12) no claim for a lipid-modifying medication.

\section{Analysis}

All study variables were analyzed descriptively. Numbers and percentages are provided for dichotomous and polychotomous variables. Means, medians, standard deviations, and percentiles are provided for continuous variables. Differences in mean values were assessed using a t-test and differences in proportions were assessed using a chi-squared test.

\section{Results}

\section{Patient Characteristics}

Of the 3.9 million health plan enrollees in 2006, lab values for LDL-C, HDL-C, and TG levels were available for a total of 529,236 patients. Of these, 65,242 (12.3\%) met the definition of having DM, and $82.3 \%(\mathrm{n}=$ 53,679 ) of the DM patients had at least 1 suboptimal lipid value and $17.7 \%(\mathrm{n}=11,563)$ had optimal values for all lipid tests The mean age of the study population was 52 years, and was similar in the suboptimal cohort and optimal cohort $(\mathrm{p}=0.0725)$. Fifty-two percent of patients were female and $47 \%$ were male in the study population (Table 1).

Among the diabetes patients in the suboptimal cohort ( $n=53,679)$, approximately 54\% $(n=28,728)$ had more than 1 lipid abnormality. The lipid abnormalities in the DM population were as follows: $23 \%$ had high LDL-C values only, $13 \%$ had low HDL-C only, $10 \%$ had elevated TG only, 10\% had high LDL-C and low HDLC, $12 \%$ had elevated LDL-C and TG, 16\% had low HDL$\mathrm{C}$ and elevated TG, and 16\% had abnormal values for all 3 lipid fractions.

\section{Treatments}

Overall, an increase in lipid-modifying therapy from the pre-index period to the post-index period was observed in DM patients with mixed dyslipidemia. In the preindex period, $68 \%$ of DM patients with suboptimal LDL$\mathrm{C}$ and HDL-C, 58\% with suboptimal LDL-C and TG, $32 \%$ with suboptimal HDL-C and TG, and 60\% with 
Table I: Demographic Information for Patients with Diabetes

\begin{tabular}{|c|c|c|c|c|c|c|c|}
\hline \multirow[t]{2}{*}{ Demographics } & \multicolumn{2}{|c|}{ Total $(\mathrm{N}=65,242)$} & \multicolumn{2}{|c|}{ Suboptimal Cohort $(N=53,679)$} & \multicolumn{2}{|c|}{ Optimal Cohort $(\mathrm{N}=1 \mathrm{I}, 563)$} & \multirow[t]{2}{*}{$\mathrm{p}$-value } \\
\hline & mean & std & mean & std & mean & std & \\
\hline \multirow[t]{2}{*}{ Age (continuous) } & 52.43 & 10.53 & 52.39 & 10.22 & 52.61 & 11.86 & 0.0725 \\
\hline & $\mathrm{n}$ & $\%$ & $\mathrm{n}$ & $\%$ & $\mathrm{n}$ & $\%$ & \\
\hline Age & & & & & & & $<0.0001$ \\
\hline$<45(\mathrm{M})$ or $<55(\mathrm{~F})$ & 22,697 & 34.79 & $19,01 \mid$ & 35.42 & 3.686 & 31.88 & \\
\hline$>=45(\mathrm{M})$ or $>=55(\mathrm{~F})$ & 42,545 & 65.21 & 34,668 & 64.58 & 7,877 & 68.12 & \\
\hline Gender & & & & & & & $<0.0001$ \\
\hline Male & 34,538 & 52.94 & 27,695 & 51.59 & 6,843 & 59.18 & \\
\hline Female & 30,704 & 47.06 & 25,984 & $48.4 I$ & 4,720 & 40.82 & \\
\hline Geographic Location & & & & & & & $<0.0001$ \\
\hline Northeast & 8,695 & 13.33 & 7,061 & 13.15 & 1,634 & 14.13 & \\
\hline Midwest & 13,374 & 20.50 & 10,979 & 20.45 & 2,395 & 20.71 & \\
\hline South & 40,440 & 61.98 & 33,489 & 62.39 & 6,951 & 60.11 & \\
\hline West & 2,733 & 4.19 & 2,150 & 4.01 & 583 & 5.04 & \\
\hline
\end{tabular}

$\%$ is calculated from the Column total.

suboptimal values for all 3 lipid parameters received no treatment for dyslipidemia. In the post-index period, the percentages of patients not receiving therapy decreased to $58 \%, 43 \%, 29 \%$, and $45 \%$ in each group, respectively. Patients with suboptimal HDL-C and TG had the smallest decrease in the percentage of patients not receiving lipid-modifying therapy (Figure 1 ).

Among DM patients with more than 1 lipid abnormality, $25 \%$ to $37 \%$ were prescribed statin monotherapy, 3-6\% received fibrate monotherapy, and less than $1 \%$ received niacin monotherapy during the post-index period. Figure 2 illustrates the pre-index versus post-index monotherapy patterns for DM patients with mixed dyslipidemia. The proportion of DM patients treated with statins in combination with other lipid-modifying drugs increased from the pre-index to post-index time period but still remained low $(1.5 \%$ to $3.5 \%$ of patients receiving statin and fibrate therapy, $<1.0 \%$ to $2.6 \%$ of those receiving statin and niacin therapy, and $7.0 \%$ to $10.4 \%$ of those receiving statin and other lipid-modifying therapy). Figure 3 shows the pre-index versus post-index combination treatment patterns for this patient group.

The treatment patterns for patients with DM were also evaluated by quartiles of suboptimal LDL-C, HDL-C, and TG values. The ranges for the quartiles are defined in Figure 4. A total of 32,855 patients $(61 \%)$ were identified as having elevated LDL-C, 29,398 (55\%) had low HDL-C, and 28,755 (54\%) had elevated TG. Among the patients with a suboptimal lipid value and DM, the percentages of patients not receiving treatment were $63 \%, 47 \%$, and $49 \%$, respectively. The percentage of patients not receiving lipid-modifying therapy decreased in the post-index period, but remained high in every quartile as illustrated in Figure 4. In the first quartile, the percentages of patients not receiving treatment for suboptimal LDL-C, HDL-C, and TG were 53\%, 36\%, and $39 \%$, respectively. In the fourth quartile, the percentage of patients not receiving therapy decreased to $43 \%$ for patients with suboptimal LDL levels, but increased for patients with suboptimal HDL-C and TG (to $46 \%$ and $40 \%$, respectively). When treatment was initiated, statin monotherapy was the most commonly prescribed regardless of the type of lipid abnormality and the quartile.



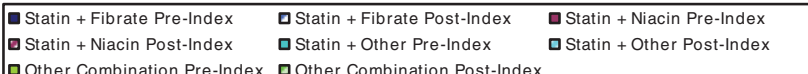

Figure I

DM Patients $(n=28,728)$ with $>$ I Suboptimal Lipid Value Not Taking Any Lipid-Modifying Therapy, Pre- vs. Post-Index. 


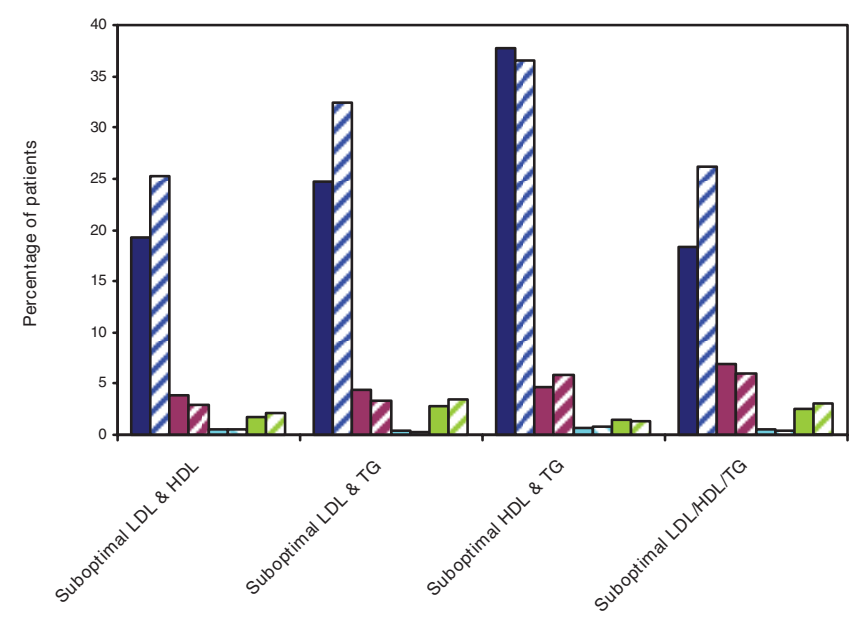



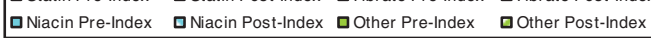

Figure 2

Monotherapy Patterns for DM Patients with > I Suboptimal Lipid Value, Pre-Index vs. Post-Index.



\begin{tabular}{|c|c|c|}
\hline \begin{tabular}{|l} 
Statin + Fibrate Pre-Index \\
a Statin + Niacin Post-Index \\
a Other Combination Pre-Index
\end{tabular} & $\begin{array}{l}\text { 口Statin + Fibrate Post-Index } \\
\text { ם Statin + Other Pre-Index } \\
\text { Q Other Combination Post-Index }\end{array}$ & $\begin{array}{l}\text { - Statin + Niacin Pre-Index } \\
\text { —Statin + Other Post-Index }\end{array}$ \\
\hline
\end{tabular}

\section{Figure 3}

Combination Therapy Patterns for DM Patients with > I Suboptimal Lipid Value, Pre-Index vs. Post-Index.

\section{Discussion}

Despite the availability of treatment guidelines for dyslipidemia management and the abundance of clinical trial evidence highlighting the benefits of lipid-modifying therapy to reduce the risk for CHD and its clinical sequelae, treatment of $\mathrm{DM}$ patients with abnormal LDL-C, HDL-C, and TG levels continues to be



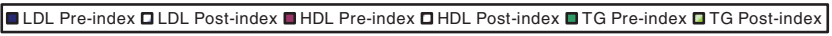

Figure 4

DM Patients Not Receiving Lipid-Modifying

Treatment, by Quartiles of LDL-C, HDL-C, and TG Value, Pre-Index vs. Post-Index.

suboptimal. In our patient population, $16 \%$ of patients with suboptimal lipid values and $21 \%$ of our mixed dyslipidemia group had DM. Our DM patient population had a high $(>50 \%)$ proportion of patients with abnormal HDL-C and TG levels. This is in line with the results of a recent study by Grant et al. [27], who also found a high prevalence of suboptimal HDL-C among patients with type 2 diabetes, with nearly half $(49.5 \%)$ of patients exhibiting low HDL-C. Another study, involving a high-risk population with documented CHD or CHD risk equivalents, also found a high prevalence of low HDL-C across various LDL-C levels, including among patients taking statins [28]. In that study, low HDL-C was most prevalent in patients with LDL-C of $70 \mathrm{mg} / \mathrm{dL}$ or lower, and was equally and highly prevalent in patients taking statins (67\%) and those not taking statins (64\%).

A large percentage of DM patients with suboptimal lipid levels in our study did not receive lipid-modifying therapy either prior to or after a suboptimal lipid value was obtained (53\% and $42 \%$, respectively). While the percentage of patients receiving lipid-modifying therapy increased in the post-index period, a substantial number of DM patients with suboptimal lipid levels did not receive any lipid-modifying therapy in the post-index period irrespective of abnormal lipid parameter, or the degree of the abnormal value. At least $30 \%$ of DM patients with suboptimal lipid levels across all mixed dyslipidemic groups were not treated with any lipidmodifying medication for at least 6 months post-index. Although lipid treatment rates increased post-index in all 
mixed dyslipidemic groups, and even though treatment rates were correlated with the number of total risk factors, abnormal lipid parameters and more severe lipid value quartiles, the incremental use of lipid-modifying medication was not as substantial as would be expected, given the compelling association between multiple lipid abnormalities and cardiovascular risk in patients with DM. Of particular note is that in DM patients with low HDL-C and elevated TG, the most common post-index lipid therapy used was statin monotherapy (in 37\% of patients), and only $17 \%$ received any niacin or fibrate therapy to target suboptimal HDL-C or TG. Grant et al. [27] described similar findings, with the vast majority of their study patients receiving statin monotherapy and a very small percentage being prescribed treatment targeting the suboptimal HDL-C level.

These results are also consistent with those reported by Klingman and colleagues [29], who evaluated data from the 1999-2000 National Health and Nutrition Examination Survey to assess the state of dyslipidemia management in the US adult population and to determine whether treatment patterns were consistent with guideline recommendations. They demonstrated that $44 \%$ of treatment-eligible adults had never been told by a physician, or any other healthcare professional, that they had dyslipidemia. Among all treatment-eligible adults, only $30 \%$ were adopting therapeutic lifestyle changes (TLC) and only $10 \%$ were receiving lipidmodifying therapy. Very high percentages of treatmenteligible adults were receiving no treatment at all for dyslipidemia: $69 \%$ overall, $61 \%$ of high-risk adults, $70 \%$ of medium-risk adults, and $77 \%$ of low-risk adults. Unlike the present study, however, Klingman and colleagues focused on the achievement of LDL-C goals and did not evaluate HDL-C and TG levels.

Although the reasons for the suboptimal treatment of dyslipidemia are beyond the scope of this study, several explanations have been proposed to account for the discrepancy between guideline recommendations and real-world treatment patterns. These include suboptimal patient-follow-up, use of low-potency statins in high-risk patients, inaccurate pill counts and refill records, patients' inability to pay for medications, lack of patient motivation [30], physician time constraints, difficulty understanding and applying NCEP guidelines [19], and lingering concerns about the potential hepatic and skeletal muscle toxicity of combinations of lipidmodifying drugs [31].

NCEP ATPIII recommends adjunctive therapy with niacin or fibrates (to achieve non-HDL goal) for dyslipidemic patients with multiple CVD risk factors who have low HDL-C and/or high TG, after the LDL-C goal has been achieved with statin monotherapy $[7,19,32]$. Our study results suggest that the majority of patients with DM receiving lipid-modifying treatment are being treated with statin monotherapy. Although it is well documented that the different classes of lipidmodifying medications have different (complementary) effects on lipid parameters [19], statin monotherapy continued to be prescribed to $81 \%$ of patients with more than 1 suboptimal lipid value who received treatment during the post-index period of our study, even though many patients with multiple lipid abnormalities - i.e., those with suboptimal HDL-C and TG levels - may have gained greater benefit from adjunctive treatment with either niacin, a drug with the greatest capacity to increase HDL-C, or fibrates, which have the most pronounced capacity to decrease elevations in serum TG. This is despite the fact that NCEP ATPIII was published in 2001, 5 years prior to the conduct of this study [19].

Our findings regarding prescribing habits for mixed dyslipidemia are consistent with results reported by Stacy and colleagues [33], who evaluated 600 highvolume prescribers of lipid-modifying drugs in 6 metropolitan areas identified from the IMS Health prescription database. Their study also found that $40 \%$ of the study population potentially had mixed dyslipidemia. These investigators reported that a high percentage of patients $(69 \%)$ were prescribed monotherapy with lipid-modifying medication, of which $82 \%$ was statin monotherapy; furthermore, 14\% were not prescribed any lipid-modifying drug therapy.

\section{Study Limitations}

The findings of this study must be considered within the limitations of the data and study design. Claims data are collected for the purpose of payment and not research and may not accurately capture a patient's medical use history. While these data provide insight into real-world treatment patterns, they are subject to possible coding errors. Certain information is not readily available in claims data that could have an effect on study outcomes, such as certain clinical and disease-specific parameters.

Furthermore, the presence of a prescription claim does not necessarily indicate that the drug was taken or that it was taken as prescribed - nor does it reflect those patients who may have received drugs without the presence of a prescription claim either by receiving drug samples or filling a prescription outside of the health care pharmacy system. The presence of a diagnosis code on a medical claim does not demonstrate positive presence of disease, as it may be incorrectly coded or included as a rule-out criterion rather than as an indication of actual disease. 
In addition, because patients were identified from a managed care plan, this study may not be generalizable to the general population and may not be applicable to a setting outside of managed care. Finally, a limitation specific to this study is that the description for values in the laboratory results does not clearly indicate whether lipid values were obtained during fasting. Although these limitations do not reduce the strength of the study, they must be considered when interpreting the results.

\section{Conclusion}

In real-world clinical practice, pharmacologic treatment rates increased only slightly upon assessment of multiple lipid abnormalities and in patients with DM. Over $42 \%$ of DM patients with mixed dyslipidemia received no lipid-modifying therapy after suboptimal lipid levels were ascertained, and those patients who were treated, even those with low HDL-C and high TG levels who may benefit from adjunctive treatment with niacin or fibrates, were primarily prescribed statin monotherapy. These study results suggest DM patients are not being treated to ADA-suggested targets. Further research is warranted to investigate how physicians tailor lipid-modifying therapy in these patients so that solutions aimed at improving treatment rates can be devised in order to help DM patients better achieve guideline-defined LDLC, HDL-C, and TG goals, thereby potentially reducing their risk for cardiovascular events.

\section{Competing interests}

DL works for Abbott Laboratories, which provided the financial support to sponsor the research for this study. PT received an honorarium from Abbott Laboratories for consulting services on this study and development of this manuscript.

\section{Authors' contributions}

DL conceived of the study and participated in its design and coordination. PT participated in the design of the study and helped draft the manuscript. JS participated in the design of the study and performed the statistical analysis and programming. VZ participated in the design and coordination of the study and drafted the manuscript. All authors read and approved the final manuscript.

\section{Acknowledgements}

The study authors would like to thank Victoria Porter, i3 Innovus, for her assistance with the preparation of this manuscript.

\section{References}

I. Moss SE, Klein R and Klein BE: Cause-specific mortality in a population-based study of diabetes. Am J Public Health I991, 81:1158-1162.

2. Huxley R, Barzi $F$ and Woodward M: Excess risk of fatal coronary heart disease associated with diabetes in men and women: meta-analysis of $\mathbf{3 7}$ prospective cohort studies. BMJ 2006, 332:73-78.

3. Grundy SM, Brewer HB Jr, Cleeman JI, Smith SC Jr and Lenfant C: For the Conference Participants. Definition of metabolic syndrome: Report of the National Heart, Lung, and Blood Institute/American Heart Association conference on scientific issues related to definition. Circulation 2004, 109:433-438.

4. Davidson MH and Toth PP: Comparative effects of lipidlowering therapies. Prog Cardiovasc Dis 2004, 47:73-104.

5. Hoang KC, Ghandehari H, Lopez VA, Barboza MG and Wong ND: Global coronary heart disease risk assessment of U.S. persons with the metabolic syndrome. Diabetes Care 2008, 3 I (7): | 1405-1409.

6. Best JD and $\mathrm{O}^{\prime} \mathrm{Neal} \mathrm{DN}$ : Diabetic dyslipidaemia: current treatment recommendations. Drugs 2000, 59: II0I-IIII.

7. Ito MK: Advances in the understanding and management of dyslipidemia: using niacin-based therapies. Am J Health Syst Pharm 2003, 60(I3 Suppl 2):SI5-S2I.

8. Bestermann W, Houston MC, Basile J, Egan B, Ferrario CM, Lackland D, Hawkins RG, Reed J, Rogers P, Wise D and Moore MA: Addressing the global cardiovascular risk of hypertension, dyslipidemia, diabetes mellitus, and the metabolic syndrome in the southeastern United States, part II: treatment recommendations for management of the global cardiovascular risk of hypertension, dyslipidemia, diabetes mellitus, and the metabolic syndrome. Am J Med Sci 2005, 329:292-305.

9. Rubins HB, Robins SJ, Collins D, Iranmanesh A, Wilt TJ, Mann D, Mayo-Smith M, Faas FH, Elam MB, Rutan GH and Department of Veterans Affairs HDL Intervention Trial Study Group: Distribution of lipids in 8,500 men with coronary artery disease. Am J Cardiol 1995, 75: I| 196-1201.

10. Genest J], McNamara JR, Salem DN and Schaefer E): Prevalence of risk factors in men with premature coronary artery disease. Am J Cardiol 1991, 67:1 I85-I I89.

II. Pekkanen J, Linn S, Heiss G, Suchindran CM, Leon A, Rifkind BM and Tyroler HA: Ten-year mortality from cardiovascular disease in relation to cholesterol level among men with and without preexisting cardiovascular disease. N Engl J Med 1990, 322:1700-1707.

12. Gordon DJ and Rifkind BM: High-density lipoprotein - the clinical implications of recent studies. N Engl J Med 1989, 32I:|3||-|3|6.

13. Goldbourt U, Yaari S and Medalie JH: Isolated low HDL-C cholesterol as a risk factor for coronary heart disease mortality: a $\mathbf{2 1}$-year follow-up of $\mathbf{8 0 0 0}$ men. Arterioscler Thromb Vasc Biol 1997, 17:107-113.

14. Rubins HB, Robins SJ, Collins D, Fye CL, Anderson JW, Elam MB, Faas FH, Linares E, Schaefer EJ, Schectman G, Wilt TJ and Wittes J: Gemfibrozil for the secondary prevention of coronary heart disease in men with low levels of high-density lipoprotein cholesterol. N Engl J Med 1999, 34 I:410-4I8.

15. Miller M, Cannon CP, Murphy SA, Qin J, Ray KK and Braunwald E: PROVE ITTIMI 22 Investigators. Impact of triglyceride levels beyond low-density lipoprotein cholesterol after acute coronary syndrome in the PROVE IT-TIMI 22 trial. Am Coll Cardiol. 2008, 5 I(7):724-730.

16. Tenkanen L, Manttari M and Manninen V: Some coronary risk factors related to the insulin resistance syndrome and treatment with gemfibrozil. Experience from the Helsinki Heart Study. Circulation 1995, 92: 1779-1785.

17. The BIP Study Group: Secondary prevention by raising HDL-Ccholesterol and reducing triglycerides in patients with coronary disease: the Bezafibrate Infarction Prevention (BIP) Study. Circulation 2000, 102:21-27.

18. Buse JB, Ginsberg HN, Bakris GL, Clark NG, Costa F, Eckel R and American Heart Association; American Diabetes Association: Primary prevention of cardiovascular diseases in people with diabetes mellitus: a scientific statement from the American Heart Association and the American Diabetes Association. Circulation 2007, I I5(I): | |4-26.

19. Third report of the National Cholesterol Education Program (NCEP) Expert Panel on Detection, Evaluation and Treatment of High Blood Cholesterol in Adults (Adult Treatment Panel III). Circulation 2002, I06:3|44-342I.

20. Vega GL, Ma PT, Cater NB, Filipchuk N, Meguro S, GarciaGarcia AB, Fonseca V, Gerstein HC, Grundy S, Nesto RW, Pignone MP, Plutzky J, Porte D, Redberg R, Stitzel KF, Stone NJ and American Heart Association; American Diabetes Association: Effects of adding fenofibrate $(200 \mathrm{mg} /$ day) to simvastatin ( 10 
$\mathrm{mg} /$ day) in patients with combined hyperlipidemia and metabolic syndrome. Am J Cardiol 2003, 91:956-960.

21. Wagner AM, Jorba $O$, Bonet $R$, Ordonez-Llanos J and Perez A: Efficacy of atorvastatin and gemfibrozil, alone and in low dose combination, in the treatment of diabetic dyslipidemia. J Clin Endocrinol Metab 2003, 88:32 I2-3217.

22. Derosa G, Cicero AE, Bertone G, Piccinni MN, Ciccarelli $L$ and Roggeri DE: Comparison of fluvastatin + fenofibrate combination therapy and fluvastatin monotherapy in the treatment of combined hyperlipidemia, type 2 diabetes mellitus, and coronary heart disease: a I2-month, randomized, double blind, controlled trial. Clin Ther 2004, 26:1599-1607.

23. Wake Forest University School of Medicine Public Health Sciences Department. ACCORD Trial Website. http://www. accordtrial.org/web/public/index.cfm.

24. Keevil JH, Cullen MW, Gangnon R, McBride PE and Stein JH: Implications of cardiac risk and low-density lipoprotein cholesterol distributions in the United States for the diagnosis and treatment of dyslipidemia: data from National Health and Nutrition Examination Survey 1999 to 2002. Circulation 2007, I I 5:1363-1370.

25. Howell EE, Wright SM, Bush DE, Chandra-Strobos $N$ and Henrikson CA: Insufficient treatment of hypercholesterolemia among patients hospitalized with chest pain. Clin Cardiol 2006, 29:259-262.

26. Waters DD: What the statin trials have taught us. Am J Cardiol 2006, 98:129-134.

27. Grant RW and Meigs JB: Prevalence and treatment of low HDL cholesterol among primary care patients with type 2 diabetes: an unmet challenge for cardiovascular risk reduction. Diabetes Care 2007, 30:479-484.

28. Alsheikh-Ali AA, Lin JL, Abourjaily P, Ahearn D, Kuvin JT and Karas RH: Prevalence of low high-density lipoprotein cholesterol in patients with documented coronary heart disease or risk equivalent and controlled low-density lipoprotein cholesterol. Am J Cardiol 2007, 100:1499-1501.

29. Klingman D, Williams SA, Benner JS, Smith TW, Ahn J and O'Donnell JC: Gauging the treatment gap in dyslipidemia: findings from the 1999-2000 National Health and Nutrition Examination Survey. Am Heart J 2005, 150:595-60I.

30. Davidson $\mathrm{MH}$ : Differences between clinical trial efficacy and real-world effectiveness. Am J Manag Care 2006, I2(I5 Suppl): S405-S4II.

31. Guthrie RM: How safe is aggressive statin therapy?. Prog Cardiovasc Nurs 2006, 21:140-145.

32. Pearlman BL: The new cholesterol guidelines. Applying them in clinical practice. Postgrad Med 2002, I I2:13-16.

33. Stacy TA and Egger A: Results of retrospective chart review to determine improvement in lipid goal attainment in patients treated by high-volume prescribers of lipid-modifying drugs. J Manag Care Pharm 2006, 12:745-75|.
Publish with BioMed Central and every scientist can read your work free of charge

"BioMed Central will be the most significant development for disseminating the results of biomedical research in our lifetime. "

Sir Paul Nurse, Cancer Research UK

Your research papers will be:

- available free of charge to the entire biomedical community

- peer reviewed and published immediately upon acceptance

- cited in PubMed and archived on PubMed Central

- yours - you keep the copyright

Submit your manuscript here:

http://www.biomedcentral.com/info/publishing_adv.asp
BioMedcentral 